Annuaire suisse de politique de développement

16 | 1997

Environnement et développement, 5 ans après Rio

\title{
Labels écologiques et commerce international : principaux aspects
}

René Vossenaar

\section{OpenEdition}

1 Journals

Édition électronique

URL : http://journals.openedition.org/aspd/809

DOI : 10.4000/aspd.809

ISSN : 1663-9669

Éditeur

Institut de hautes études internationales et du développement

Édition imprimée

Date de publication : 1 mars 1997

Pagination : 215-228

ISSN : 1660-5934

\section{Référence électronique}

René Vossenaar, «Labels écologiques et commerce international : principaux aspects ", Annuaire suisse de politique de développement [En ligne], 16 | 1997, mis en ligne le 08 août 2012, consulté le 08 septembre 2020. URL : http://journals.openedition.org/aspd/809 ; DOI : https://doi.org/10.4000/aspd. 809

(c) The Graduate Institute I Geneva 


\title{
LABELS ÉCOLOGIQUES ET COMMERCE INTERNATIONAL : PRINCIPAUX ASPECTS
}

\author{
RENÉ VOSSENAAR ${ }^{1}$
}

\section{INTRODUCTION}

\section{A. GÉNÉRALITÉS}

Les labels écologiques visent à promouvoir la fabrication et la consommation de produits particulièrement respectueux de l'environnement en fournissant aux consommateurs des informations relatives à l'impact environnemental de ces produits ; ces informations se rapportent en général au cycle de vie du produit. Le présent article met en évidence l'ambivalence de ces labels : si leurs objectifs sont écologiques, ils peuvent cependant se traduire par une discrimination envers les producteurs étrangers et constituer ainsi des barrières commerciales non tarifaires.

L'attribution de ce type de labels touche de plus en plus certains pays en développement, car ils exportent des produits qui appartiennent aux nouvelles catégories où les labels écologiques tendent à s'imposer. De plus, dans le cas de ces catégories de produits, on accorde parfois plus d'importance à l'impact écologique de la fabrication du produit qu'à celui de la consommation et du traitement des déchets. Les critères d'attribution concernent ainsi avant tout les matières premières et les procédés de fabrication. Or, les fabricants étrangers ne peuvent pas toujours respecter les exigences requises.

Nous examinons ci-après l'impact environnemental et, surtout, commercial des labels écologiques sur les pays en développement, en passant en revue leurs principaux aspects qui font actuellement l'objet de vifs débats dans divers organismes, y compris au sein de la Conférence des Nations unies sur le commerce et le développement (CNUCED). Notre étude se fonde largement sur les rapports élaborés par le secrétariat de la CNUCED pour faciliter les délibérations sur les labels écologiques au sein du Groupe de travail spécial sur le commerce, l'environnement et le développement. ${ }^{2}$

\section{B. PRINCIPES DE LA CERTIFICATION ÉCOLOGIQUE}

Nous n'abordons ici que les labels écologiques accordés par un tiers à des produits qui respectent certains critères fixés à l'avance, ou labels de « type I » selon la classification appliquée par l'Organisation internationale de standardisation (ISO). A l'heure actuelle, on dénombre plus de 20 programmes d'attribution de labels du type I (voir tableau 1).

\footnotetext{
${ }^{1}$ L'article est tiré de l'ouvrage : Eco-Labelling and International Trade, Edited by Simonetta Zarrilli, Veeva Jha and René Vossenaar, 1997, Macmillan publisher. Les opinions exprimées ici sont celles de l'auteur et ne reflètent pas toujours celles de la CNUCED.

2 Voir CNUCED, TD/B/WG.6/2 du 6 octobre 1994 et TD/B/WG.6/5 du 28 mars 1995.
} 
Tableau no 1

Tableau synoptique des programmes de certification

\begin{tabular}{llc}
\hline Pays / groupe de pays & Nom du programme / du label & Date de lancement \\
\hline Allemagne & Ange bleu & 1977 \\
\hline Canada & Programme choix environnemental & 1988 \\
\hline Japon & EcoMark & 1989 \\
\hline Pays nordiques & White Swan & 1989 \\
\hline Etats-Unis & Green Seal & 1989 \\
\hline Suède & Good Environmental Choice & 1990 \\
\hline Nouvelle-Zélande & Environmental Choice & 1990 \\
\hline Inde & Ecomark & 1991 \\
\hline Autriche & Label écologique autrichien & 1991 \\
\hline Australie & Environmental Choice & 1991 \\
\hline République de Corée & Ecomark & 1992 \\
\hline Singapour & Green Label Singapour & 1992 \\
\hline France & NF - Environnement & 1992 \\
\hline Pays-Bas & Stichting Milieukeur & 1992 \\
\hline Union européenne & Fleur européenne & 1992 \\
\hline Croatie & Ecologique & 1993 \\
\hline
\end{tabular}

Source : CNUCED, TD/B/WG.6/2, encadré 2.

L'attribution d'un label écologique se fonde en principe sur une approche globale qui tient compte de multiples critères ainsi que du cycle de vie des produits, dans le but d'informer le consommateur d'une réduction réelle de l'agression environnementale et non pas seulement d'un transfert des impacts sur d'autres biens environnementaux ou sur d'autres étapes du cycle de vie du produit. Dans la pratique toutefois, l'analyse du cycle de vie varie grandement d'un programme à l'autre. Les programmes de l'Union européenne (UE) et des Pays-Bas commencent par exemple par identifier les aspects écologiques prépondérants sur la base d'une matrice incluant les impacts environnementaux du produit à chacun des stades de son cycle de vie. Des critères spécifiques sont ensuite élaborés pour chacun de ces impacts. Pour ce qui est de l'analyse du cycle de vie, c'est sans doute le programme français qui est le plus rigoureux. Il arrive toutefois que les critères d'autres programmes ne se fondent que sur certains impacts environnementaux et ne prennent en compte qu'une partie du cycle de vie du produit. Les labels allemand et japonais ne considèrent par exemple qu'un seul aspect écologique qui ne concerne en général que les phases utilisation et élimination de la vie d'un produit.

Bien que l'analyse du cycle de vie constitue une approche utile du point de vue environnemental, son application systématique peut engendrer des difficultés d'ordre pratique et conceptuel, notamment dans le cadre du commerce international. Le simple fait de devoir réunir un grand nombre d'informations pour procéder à une telle analyse risque de poser des problèmes pratiques dans le cas de produits ou de matières importés. De plus, certains critères relatifs aux procédés 
et aux méthodes de production - basés sur des situations et des priorités propres au pays importateur - peuvent perdre de leur pertinence dans d'autres pays, notamment dans les pays en développement où la situation et les priorités environnementales sont souvent différentes.

\section{EFFETS DISCRIMINATOIRES POUR LES PRODUCTEURS ÉTRANGERS}

Les difficultés que les fournisseurs étrangers rencontreront peut-être au cours de la procédure d'obtention d'un label écologique correspondent en quelque sorte à celles qui désavantagent d'ordinaire un exportateur face à un producteur national. Il n'en reste pas moins que certains aspects de la certification écologique, tels que la prise en considération du cycle de vie complet d'un produit, peuvent sensiblement accroître ces difficultés. On craint en effet que, bien que les mêmes critères d'attribution des labels s'appliquent aux fournisseurs étrangers et nationaux, la procédure d'obtention ne constitue une discrimination de facto contre les producteurs étrangers, notamment ceux des pays en développement. Divers facteurs peuvent engendrer de tels effets discriminatoires :

La certification écologique tend à se fonder sur des priorités et des techniques environnementales qui prévalent dans le pays importateur, au risque de négliger des produits et des procédés de fabrication acceptables dans le pays de provenance du produit. De plus, les critères appliqués sont rarement assez souples pour refléter la situation et les priorités environnementales propres aux pays de production.

La définition de catégories de produits ainsi que la fixation de critères et de valeurs-limites peuvent favoriser les producteurs nationaux au détriment des producteurs étrangers. Les critères d'attribution se réfèrent en effet parfois à des technologies auxquelles les entreprises nationales ont plus facilement accès.

L'attribution d'un label écologique peut exiger des producteurs étrangers de se conformer à des critères qui ne jouent pas un rôle prépondérant dans leur propre pays. ${ }^{3}$ Pour obtenir un label, une entreprise serait par exemple obligée d'importer des technologies destinées à lutter contre des polluants qui jouent un grand rôle dans le pays importateur, mais qui sont de moindre importance dans le pays exportateur. $^{4}$

${ }^{3}$ On pourrait, par exemple, fixer des exigences relatives aux émissions de dioxyde de soufre pendant la production. Dans un pays où ces émissions ne suscitent pas d'inquiétude (les niveaux peuvent être très faibles par rapport à la capacité d'absorption de l'environnement), de telles exigences engendreraient des coûts supplémentaires sans améliorer pour autant l'état de l'environnement. (Voir à ce propos A. Markandya, «Eco-labelling : An Introduction and a Review ", in : Eco-labelling and International Trade, op. cité..)

${ }^{4}$ L'Union européenne peut par exemple fonder ses exigences sur les rejets de dioxyde de soufre et d'oxydes d'azote, puisque ces polluants jouent un rôle important en Europe et que des technologies ont été développées pour réduire leurs émissions. Or, les pays en développement, où ces polluants sont moins fréquents, ne se sont pas dotés de ces technologies. Pour obtenir un label écologique, les producteurs de ces pays seraient donc obligés d'importer les technologies en question. 
Les infrastructures environnementales peuvent varier grandement d'un pays à l'autre (par exemple stations d'épuration des eaux usées ; usines de traitement des déchets solides ; installations de recyclage). ${ }^{5}$

Les producteurs étrangers, en particulier dans les pays en développement, ne peuvent pas toujours s'assurer un approvisionnement suffisant en produits chimiques et autres matières admis dans la fabrication d'articles portant un label écologique. Les effets discriminatoires peuvent également toucher les fournisseurs étrangers de matières servant à la fabrication de produits certifiés « écologiques ".

Certains paramètres utilisés pour calculer l'impact sur l'environnement des produits au cours de leur cycle de vie se fondent parfois sur des informations recueillies dans le pays d'importation ou dans des pays où la situation environnementale est semblable, et peuvent, de ce fait, surestimer l'impact sur l'environnement du pays producteur. Les paramètres servant à évaluer l'énergie nécessaire à la fabrication de produits ne reflètent par exemple pas toujours la situation dans le pays du producteur.

\section{EFFETS SUR LE COMMERCE ET LA COMPÉTITIVITÉ}

L'utilisation d'un label écologique étant totalement volontaire, les entreprises exportatrices ont le choix entre demander l'attribution du label (orienter leur compétitivité sur des facteurs non financiers) ou vendre des produits sans label (se fonder sur des critères financiers). Les labels écologiques tendent ainsi à promouvoir la distinction des produits en fonction de leur qualité environnementale et peuvent avoir des conséquences sur la compétitivité.

Au moment de la rédaction du présent article, aucun producteur d'un pays en développement n'utilisait un label écologique du type I pour commercialiser ses produits dans un pays de l'OCDE. Bien que nous ne disposions d'aucune donnée sur les demandes de label refusées, on peut admettre que les producteurs des pays en développement n'ont pas encore déposé de telles demandes. Les labels écologiqes ont toutefois peut-être déjà eu des effets sur leurs activités : premièrement, ces labels ont pu diminuer la compétitivité de produits non certifiés sur des marchés spécifiques. Les importations norvégiennes de papier fin provenant du Brésil ont ainsi sensiblement diminué après l'introduction d'un label écologique. ${ }^{6}$ Deuxièmement, les labels écologiques ont pu avoir des conséquences pour les fournisseurs de matières utilisées dans la fabrication de produits portant un label. Mais il est difficile d'évaluer leur impact. Il n'en reste pas moins que les principales inquiétudes exprimées par les exportateurs brésiliens de pâte à papier récemment confrontés à l'introduction d'un label écologique européen pour le

5 Les différences que présentent les infrastructures environnementales d'un pays à l'autre se retrouvent largement dans la définition d'un « produit propre ». Ainsi, lorsqu'une station d'épuration des eaux communale au Danemark dispose d'un système de précipitation du phosphore, on préférera l'usage du phosphore à d'autres composants capables de se lier au calcium. Cette remarque vaut aussi pour les cendres volantes. Certains pays ont mis en place l'infrastructure nécessaire permettant d'utiliser ces cendres comme matière première dans les cimenteries (au Danemark, presque toutes les cendres volantes sont utilisées dans la production de ciment). Dans ces pays, on évalue ainsi différemment certaines activités qui produisent des cendres volantes que dans d'autres pays. (Voir Helle Petersen, « A Possible (International) Implementation Strategy for Product Oriented Environmental Policy Measures », in International Workshop on Product Oriented Environmental Policy, Workshop Proceedings, The Hague, du 30 septembre au 1er octobre 1993).

6 CNUCED, TD/B/40 (1)/6 du 6 août 1993. 
papier de soie se fondaient justement sur ces effets indirects. Par ailleurs, la certification écologique dans l'industrie de la chaussure pourrait avoir des conséquences fâcheuses sur les tanneries des pays en développement.

Les effets des labels écologiques sur les pays en développement dépendent essentiellement du choix des catégories de produits ouvertes à la certification, de la taille relative du marché réservée aux produits certifiés, ${ }^{7}$ des coûts de mise en conformité (y compris le coût des essais et des procédures de vérification), en relation avec d'éventuels avantages au niveau du prix pour les produits certifiés, et des coûts administratifs liés à l'attribution d'un label (redevance par exemple). Dans certains cas, ces conséquences toucheront plus les fournisseurs de matières premières plutôt que le fabricant des produits inclus dans un programme de certification.

Jusqu'ici, les labels écologiques n'ont peut-être pas eu de graves conséquences pour les pays en développement, car les produits admis dans les programmes de certification n'occupent pas une place prépondérante dans le commerce extérieur de ces pays (sauf peut-être pour ce qui est du papier et des produits à base de papier). Cependant, des propositions visant à introduire un label écologique pour le bois et les produits en bois soulèvent de vives inquiétudes dans certains pays en développement. Par ailleurs, les effets des labels écologiques commencent à se faire sentir dans d'autres secteurs d'exportation de ces pays.

L'Union européenne est ainsi sur le point de mettre en place des labels écologiques pour les chaussures et certains produits textiles (T-shirts et linge de lit). Aux Pays-Bas, le «Stichting Milieukeur » a déjà fixé des critères s'appliquant aux chaussures dans le cadre du programme national de certification écologique. En 1991, 91\% au moins de toutes les chaussures vendues aux Pays-Bas étaient importées (84\% au moins des chaussures en cuir et $97 \%$ des chaussures fabriquées à partir d'autres matériaux). Les pays en développement représentaient $35 \%$ du total des importations et $82 \%$ des importations extracommunautaires en 1993. A l'occasion d'une récente réunion du Groupe de travail spécial sur le commerce, l'environnement et le développement de la CNUCED, les représentants des pays en développement se sont déclarés inquiets face à cette tendance et s'interrogent sur l'utilité de fixer des critères de certification écologique pour des produits importés, notamment lorsque ces critères concernent l'utilisation de certains matériaux ou les procédés et les méthodes de production.

Les études de cas que nous connaissons permettent de se faire une idée des principaux facteurs qui déterminent le coût de la mise en conformité dans les catégories de produits occupant une grande place dans les exportations des pays en développement. Relevons toutefois que, puisque les producteurs des pays en développement n'ont pas encore déposé leurs demandes d'attribution de labels écologiques, ces études ne se fondent pas sur une analyse détaillée des coûts des

\footnotetext{
7 On dit souvent que les labels écologiques ne s'appliqueront qu'à une petite part du marché d'une certaine catégorie de produits. Certains programmes de certification fixent en effet des seuils élevés pour limiter le nombre des produits d'une catégorie qui se verront attribuer le label écologique dans un premier temps. Au Canada, le Programme choix environnemental se basait sur un objectif de $20 \%$ environ du marché. D'autres programmes, en revanche, tels l'EcoMark au Japon, fixent leurs critères sans tenir compte du nombre d'entreprises qui peuvent s'y conformer et une plus grande proportion d'entreprises obtiennent le label. Dans la mesure où les programmes de certification écologique tiennent de plus en plus compte du marché, il est difficile de prétendre que les effets de ces labels resteront insignifiants en arguant que les parts de marché occupées par les produits « écologiques » demeureront réduites.
} 
adaptations nécessaires, mais plutôt sur une première évaluation des difficultés que les entreprises pourraient rencontrer si elles désirent respecter différents critères (dans le cas d'une demande d'attribution d'un label). Ces études se fondent sur des entretiens avec des producteurs, des exportateurs et des représentants d'associations industrielles. ${ }^{8}$

Ces études montrent qu'il est possible, aux fins d'analyse, de classer les effets potentiels des systèmes de certification écologique sur la compétitivité des pays en développement dans trois grandes catégories : (i) coût des matières ; (ii) coûts d'investissement ; (iii) coût des essais et des procédures de vérification. Elles ont de plus démontré que, dans chacune de ces catégories, les effets potentiels varient grandement selon la taille de l'entreprise. Les petites et moyennes entreprises ne subissent ainsi pas les mêmes conséquences que les grandes compagnies.

Reste à savoir si, en visant une niche de marché potentielle réservée à des produits portant un label écologique, les entreprises pourraient - grâce à des différences de prix et / ou en occupant une part de marché plus grande - couvrir les dépenses consenties pour adapter leurs procédés de production ainsi que la qualité des matières premières aux critères d'un système de certification. Il n'est toutefois pas toujours possible de réaliser des gains considérables sur les différences de prix, de sorte que les dépenses additionnelles consenties en vue d'obtenir l'attribution d'un label écologique recèlent un risque économique indéniable.

\section{EFFETS SUR L'ENVIRONNEMENT}

Des mesures qui visent à informer le consommateur sur les avantages écologiques d'un produit et qui le poussent à prendre conscience des problèmes environnementaux, tels les labels écologiques, peuvent susciter - du côté de la demande - des pressions propices à l'innovation et contribuent ainsi à réduire l'impact sur l'environnement de la production et de la consommation. Il est toutefois difficile de déterminer dans quelle mesure la certification écologique a déjà contribué à réduire les agressions environnementales. En effet, s'il existe bel et bien des éléments qui prouvent les effets bénéfiques pour l'environnement de certains labels, ils se limitent à des cas particuliers. Quelques années après l'introduction de labels écologiques pour les appareils de chauffage au mazout et au gaz dans le cadre du programme allemand «Ange bleu», les émissions de dioxyde de soufre, de monoxyde de carbone et d'oxydes d'azote ont par exemple diminué de plus de $30 \%$, tandis que le rendement énergétique de ces appareils augmentait sensiblement. L'introduction d'un label écologique a également fait passer la part de marché des peintures et vernis à faible teneur en solvant de 1 à $50 \%$ et l'on estime que la quantité de solvants rejetés dans l'environnement a diminué de 40000 tonnes environ dans le même temps. ${ }^{9}$

${ }^{8}$ Les producteurs des pays en développement tendent à fonder leur compétitivité exclusivement sur le facteur prix et s'intéresseront peut-être très peu aux labels écologiques. Les producteurs de pays plus avancés pourront s'efforcer d'accroître la qualité de leurs produits dans le but de les écouler sur des marchés préférentiels. Or les labels écologiques pourraient avoir un effet négatif sur ces efforts.

9 Voir Hartwell R. V. et Bergkamp L., «Eco-labelling in Europe : New Market-related Environmental Risks ? », BNA International Environmental Daily, 20 octobre 1992 ; et Porter M. et van der Linde C., " Green and Competitive », Harvard Business Review, septembre-octobre 1995, p. 120-134. 
Jusqu'à présent, aucune étude systématique n'a hélas été consacrée à l'évaluation des effets sur l'environnement des programmes actuels de certification écologique. Il semblerait que ces effets dépendent dans une large mesure de l'applicabilité et de l'importance des critères de certification, ainsi que de la part de marché détenue par les produits portant un label écologique ; celle-ci étant elle-même déterminée par la préférence des consommateurs pour les produits « propres » et par la capacité d'adaptation des producteurs et des fournisseurs. Pour que la certification écologique devienne un instrument de commercialisation efficace, il faut donc, d'une part, que le public sache qu'il existe des programmes de certification et des produits portant un label écologique et, d'autre part, que les producteurs acceptent de mettre sur le marché un volume important de produits certifiés.

Tableau no 2

Nombre de catégories de produits et de produits visés par différents programmes de certification écologique

\begin{tabular}{|c|c|c|c|c|c|}
\hline & \multirow{2}{*}{$\begin{array}{l}\text { Catégories } \\
\text { de produits } \\
\text { pour lesquels } \\
\text { des critères } \\
\text { sont établis }\end{array}$} & \multicolumn{3}{|c|}{$\begin{array}{l}\text { Labels écologiques } \\
\text { attribués }\end{array}$} & \multirow[b]{2}{*}{$\begin{array}{l}\text { A des } \\
\text { produits }\end{array}$} \\
\hline & & $\begin{array}{l}\text { A des } \\
\text { catégories } \\
\text { de produits }\end{array}$ & $\begin{array}{l}\text { A des } \\
\text { fabricants }\end{array}$ & $\begin{array}{l}\text { Dont } \\
\text { fabricants } \\
\text { étrangers }\end{array}$ & \\
\hline Canada & 31 & 15 & 116 & 17 & 1500 \\
\hline Union européenne & 5 & 1 & 1 & & 8 \\
\hline Allemagne & 81 & 61 & 1058 & 175 & 4353 \\
\hline Japon & 65 & 63 & 1039 & 22 & 2322 \\
\hline Pays-Bas & 20 & 4 & 10 & 3 & 40 \\
\hline Pays nordiques & 31 & 15 & 182 & 19 & \\
\hline
\end{tabular}

Source : CNUCED, TD/B/WG.6/5, encadré 1 .

Analyser la prise de conscience des consommateurs et la réaction des producteurs peut constituer un instrument utile pour examiner les effets potentiels des labels écologiques sur l'environnement. Les consommateurs connaissent de plus en plus les programmes de certification écologique et il ressort de divers sondages que nombre d'entre eux sont prêts à payer un prix plus élevé pour des produits écologiques.

Les préoccupations des consommateurs peuvent toutefois se focaliser sur un problème précis tel que le recyclage et la déforestation. Les labels écologiques pourraient donc jouer un rôle important dans les secteurs des produits en bois ou à base de papier, alors qu'ils risquent de rester peu efficaces dans d'autres secteurs dont les consommateurs se préoccupent moins. Par ailleurs, les acheteurs institutionnels constituent aussi un marché potentiel pour les produits écologiques. En effet, certains pays de l'OCDE encouragent la prise en compte d'impératifs écologiques dans le cadre des achats publics, bien qu'ils ne favorisent en général pas expressément les produits portant un label écologique. 
L'expérience montre qu'il est difficile de créer des labels écologiques capables de susciter l'enthousiasme parmi les producteurs. Le Programme choix environnemental du Canada a, par exemple, fixé des critères pour 31 catégories de produits, mais les labels ne sont utilisés que dans 15 d'entre elles (voir tableau 2.). De plus, la majeure partie des recettes dégagées par le programme au titre de l'octroi de licences provient de deux catégories de produits seulement : la peinture et le papier fin. Les responsables canadiens ont toutefois décidé de lancer un plan d'action visant à élargir la présence des produits écologiques sur le marché. ${ }^{10}$

Les programmes les plus efficaces - pour ce qui est du nombre de produits portant des labels écologiques - sont ceux de l'Allemagne (Ange bleu) et du Japon (EcoMark). ${ }^{11}$ Cependant, un nombre limité de catégories réunissent la moitié environ des produits écologiques en Allemagne. Il s'agit notamment des revêtements peu polluants, ainsi que de produits à base de papier et de carton recyclés.

\subsection{EFFETS SUR LES PAYS EN DÉVELOPPEMENT}

En théorie, les systèmes de certification écologique adoptés par des pays de l'OCDE peuvent avoir des effets positifs sur l'environnement et le commerce des pays en développement, mais ces effets resteront sans doute modestes. Certaines grandes entreprises établies dans les pays en développement, qui disposent des moyens financiers et technologiques nécessaires pour investir dans des améliorations environnementales, pourront remplir les conditions requises pour obtenir un label écologique, ce qui leur permettra de vendre leurs produits sur des marchés préférentiels. Toutefois, comme nous l'avons indiqué plus haut, les coûts additionnels de l'adaptation aux critères de certification recèlent un certain risque économique.

L'une des questions essentielles est de savoir si les adaptations nécessaires pour obtenir un label écologique sur les marchés d'exportation se justifient également au regard des conditions économiques, technologiques et écologiques du pays producteur. Des critères de certification écologique à la fois objectifs, compréhensibles et réalistes, sont davantage susceptibles d'inciter les producteurs des pays en développement à concevoir et à fabriquer des produits répondant à ces mêmes critères.

Un certain nombre de pays en développement et de pays en transition ont déjà mis sur pied leurs propres programmes de certification écologique (Inde, République de Corée et Singapour, par exemple) ou sont en passe de le faire. Tout comme dans les pays industrialisés, ces programmes ont pour but d'améliorer la situation environnementale en fournissant des informations aux consommateurs

\footnotetext{
${ }^{10}$ Les responsables du programme espèrent accroître de 60 le nombre des catégories de produits auxquelles des labels écologiques pourront être attribués. A cet effet, le programme met l'accent sur l'orientation vers le marché, sur la sensibilisation du public (grâce à une coopération avec les titulaires de licences dans le domaine de la publicité et du marketing) et sur une nouvelle approche des critères de certification. A cet effet, les responsables ont commandé une étude pour recenser les catégories de biens et de services qu'il serait possible d'intégrer dans le programme. Ces biens et services ont été classés par ordre de priorité en fonction de leurs avantages environnementaux, du volume de leurs ventes (compte tenu des dépenses des ménages et des marchés publics) et de la réaction éventuelle des professionnels du secteur concerné.

${ }^{11}$ Dans ces deux programmes, les critères d'attribution du label écologique mettent plutôt l'accent sur les phases que constituent l'utilisation et l'élimination dans le cycle de vie des produits (voir CNUCED, TD/B/WG.6/2, encadré 4).
} 
et en encourageant les producteurs à adopter des procédés de production plus respectueux de l'environnement. Les labels écologiques et le processus d'éducation connexe peuvent inciter le consommateur à prendre mieux conscience des problèmes liés à l'environnement.

Le marché intérieur des produits portant un label écologique étant généralement étroit, les principaux objectifs consistent souvent à conserver les marchés d'exportation et à améliorer la compétitivité des produits vendus à l'étranger. Pour être plus efficaces, les programmes de certification écologique cherchent à instituer une certaine reconnaissance mutuelle avec d'autres programmes similaires dans les pays de l'OCDE.

\section{PRISE EN COMPTE DES INTÉRÊTS DES PAYS EN DÉVELOPPEMENT}

Nous avons expliqué plus haut pourquoi les programmes de certification écologique suscitent des appréhensions parmi les producteurs des pays en développement. D'un point de vue commercial, ces programmes risquent en effet de nuire à la compétitivité des exportations et de constituer un obstacle non tarifaire au commerce. Du point de vue de l'environnement, on craint que les critères de certification qui répondent aux problèmes écologiques locaux et aux priorités des pays industrialisés se révèlent inopportuns ou inadaptés dans d'autres pays, notamment dans les pays en développement.

Pour tenir compte des intérêts des pays en développement dans la mise au point de programmes de certification écologique, il faut commencer par améliorer la transparence et par permettre à ces pays de participer à l'élaboration des critères applicables aux produits qui occupent une place importante dans leurs exportations. Dans ce domaine, l'aide technique peut jouer un rôle déterminant. Il serait également utile de mettre en place des principes directeurs, que l'ISO développe en ce moment, ainsi que d'analyser les liens qui existent entre la certification écologique et les règles et principes de l'OMC. A long terme, la reconnaissance mutuelle et l'admission par les organismes de certification écologique des pays industrialisés de critères environnementaux certes différents mais "équivalents », qui tiennent compte de la situation environnementale dans les pays en développement, pourraient également servir les intérêts de ces pays.

\subsection{TRANSPARENCE}

La certification écologique suit une procédure relativement transparente au niveau national, mais elle n'offre pas toujours aux producteurs étrangers la possibilité d'exprimer leur avis et leurs préoccupations. Cette situation s'explique en partie par le fait que la certification écologique était, à l'origine, un instrument de la politique intérieure visant la protection de l'environnement et que nul n'avait supposé qu'elle aurait des effets notables sur le commerce. Les effets potentiels des labels écologiques sont dans une certaine mesure similaires à ceux de normes et de réglementations techniques. C'est pourquoi l'expérience fournie par l'application de l'Accord relatif aux obstacles techniques au commerce de l'OMC pourrait être utilisée pour limiter les effets pervers des labels écologiques. Les réglementations de l'OMC pourraient toutefois se révéler moins adaptées à 
d'autres aspects de cette certification, tels que l'analyse du cycle de vie et la mise en œuvre de critères fondés sur les procédés et les méthodes de production.

Il importe donc d'analyser les exigences qui pourraient améliorer la transparence des programmes de certification écologique, ainsi que d'envisager les moyens d'y parvenir. Il serait par ailleurs utile d'évaluer ces exigences aux divers stades du processus de certification, notamment dans le cadre (i) de la sélection des produits et de la formulation des critères et (ii) de la phase de consultation du public. Les procédures de certification écologique sont généralement ouvertes au public, de sorte que les travaux des organes compétents sont ouverts à divers groupes d'intérêts, mais il s'agit en général de groupes locaux. Les producteurs étrangers ne peuvent en principe pas participer directement au choix des produits, ni à la formulation des critères. Ils doivent donc s'en remettre à la procédure publique pour présenter leurs vues et leurs préoccupations. ${ }^{12}$

\subsection{ASSISTANCE TECHNIQUE}

L'assistance technique et le renforcement des capacités dans le domaine des labels écologiques et de la certification peuvent contribuer à réduire les éventuelles conséquences négatives des labels écologiques pour les pays en développement et aider les producteurs à tirer parti de nouveaux débouchés commerciaux pour les produits écologiques. Une assistance technique visant à renforcer les capacités pourrait également aider les pays en développement à mettre en place leurs propres systèmes de certification écologique et contribuer à promouvoir la reconnaissance mutuelle de ces programmes.

Une assistance technique s'impose en outre pour permettre aux pays en développement de participer véritablement aux futurs débats internationaux sur les labels écologiques, en particulier au sein de l'ISO. De plus, la création dans les pays en développement et dans les pays en transition d'organismes de normalisation - ou la formation des organismes existants - chargés de mener à bien les procédures d'essai et de certification dans le pays producteur, aurait pour conséquence de réduire les coûts induits par la certification écologique.

\subsection{PROCÉDÉS ET MÉTHODES DE PRODUCTION}

L'application aux produits importés de critères particuliers liés aux procédés et aux méthodes de production pose souvent un certain nombre de problèmes, notamment lorsque les produits proviennent de pays où la situation de l'environnement et l'état du développement diffèrent beaucoup de ceux du pays importa-

\footnotetext{
${ }^{12}$ La plupart des programmes de certification écologique prévoient un examen public des projets de critères avant la publication des critères définitifs. Toute partie intéressée, $y$ compris les producteurs étrangers, peut s'en prévaloir pour faire connaître ses vues. Les conditions de transparence des programmes de certification écologique existants ne diffèrent donc pas sensiblement des dispositions en matière de transparence figurant dans l'Accord sur les obstacles techniques au commerce. Toutefois, la participation de producteurs étrangers à cet examen dépend de nombreux facteurs, dont la diffusion en temps voulu de l'information relative aux nouvelles catégories de produits auxquelles on envisage d'appliquer le programme de certification, la durée du processus d'examen et la capacité d'être physiquement présent et de consacrer suffisamment de temps à cet examen. Il importerait donc d'améliorer la procédure de consultation lorsque les importations jouent un rôle important, par exemple grâce à un système de notification plus performant.
} 
teur. Les programmes de certification écologique ne retiennent d'ailleurs pas tous ce type de critères.

On prétend souvent qu'une bonne information du consommateur passe nécessairement par l'analyse du cycle de vie et par les critères liés aux procédés et aux méthodes de production. Reste à savoir dans quelle mesure des labels écologiques qui ne prennent pas en compte la situation spécifique de l'environnement du pays producteur fournissent l'information requise à ce même consommateur.

L'une des solutions consisterait à éviter les critères liés aux procédés et aux méthodes de production dans le cas de catégories de produits fabriqués essentiellement dans les pays en développement. Si l'on souhaite malgré tout appliquer ce type de critères, diverses solutions peuvent être envisagées - après un examen au cas par cas - pour éviter que le programme n'engendre des effets pervers pour les partenaires commerciaux. Il est en effet parfois préférable d'exempter les producteurs étrangers de l'obligation de respecter certains critères de fabrication. ${ }^{13} \mathrm{Il}$ est également possible de limiter l'exigence imposée aux produits provenant de pays en développement de respecter les règles en vigueur dans le pays de production. Notons ici que les règlements environnementaux des pays en développement sont parfois relativement sévères, mais que leur application se heurte souvent à des difficultés. Les labels écologiques pourraient dès lors remplir une fonction utile en encourageant les producteurs à respecter les normes environnementales existantes.

Une autre approche consisterait à faire preuve d'une grande prudence lors de l'élaboration de critères liés aux procédés et aux méthodes de production pour des secteurs où les importations sont prédominantes. ${ }^{14}$ On éviterait ainsi de fixer des critères et des valeurs limites trop contraignantes pour des catégories de produits comprenant une importante proportion d'importations, provenant notamment de pays en développement, ou on les fixerait de telle façon que les pays producteurs n'aient pas à en souffrir. Dans d'autres cas, des notions telles que les « équivalences » et la « reconnaissance mutuelle »-traitées ci-après - pourraient se révéler fort utiles pour résoudre le problème des critères liés aux procédés et aux méthodes de production.

\subsection{EQUIVALENCES ET RECONNAISSANCE MUTUELLE}

Dans le cadre de la certification écologique, la notion d'équivalence signifie que, lorsque des objectifs environnementaux comparables peuvent être atteints de multiples façons, compte tenu de la situation environnementale propre à chaque pays, différents critères peuvent être acceptés aux fins de l'attribution de labels écologiques. Ce principe pourrait s'appliquer dans deux contextes différents. Dans le premier, le programme de certification écologique du pays importateur considère le respect de certaines exigences écologiques ou l'adoption de cer-

\footnotetext{
${ }^{13}$ Le label écologique hollandais pour les chaussures, par exemple, exempte les producteurs étrangers de l'obligation de traiter les déchets solides contenant du chrome (par recyclage, mise en décharge sûre ou incinération permettant de récupérer le chrome) si ces déchets ne sont pas considérés comme des déchets chimiques dans le pays du producteur.

14 Programme Choix environnemental, Environnement Canada, « Eco-labelling and PPMs : The international Context ». Document présenté à l'atelier de l'OCDE sur les labels écologiques et le commerce international, Londres, 6 et 7 octobre 1994
} 
taines mesures visant à améliorer l'état de l'environnement comme « équivalent » à l'observation des critères et seuils du pays importateur, même lorsqu'il n'existe aucun programme de certification écologique dans le pays exportateur. Dans le second, la notion de norme « équivalente » est généralement considérée comme une condition sine qua non de la reconnaissance mutuelle des programmes de certification écologique.

En se penchant sur la question des équivalences, il convient de distinguer les critères liés aux produits et ceux qui visent les procédés de fabrication. Les premiers tiennent compte des effets d'un produit sur l'environnement du pays importateur durant les phases de consommation et d'élimination. La possibilité de fixer des critères « équivalents » dans ce domaine est en général assez limitée, en comparaison avec le potentiel qu'offrent les critères liés aux procédés et aux méthodes de production. L'environnement du pays importateur n'étant pas affecté par des procédés et des méthodes qui relèvent de problèmes environnementaux du pays exportateur, il est tout à fait possible de considérer comme équivalents des critères écologiques qui correspondent aux conditions et aux priorités environnementales de ce dernier.

Jusqu'à présent, le principe d'équivalence n'a été appliqué que dans le contexte de mesures relatives aux produits. Il est question de normes « équivalentes » tant dans l'Accord sur les obstacles techniques au commerce (Code de la normalisation) ${ }^{15}$ que dans l'Accord sur l'application des mesures sanitaires et phytosanitaires (Accord SPS), ${ }^{16}$ négociés dans le cadre du Cycle d'Uruguay. Ces textes semblent reconnaître que certains « objectifs » ou « niveaux de protection appropriés » peuvent être atteints au moyen de normes différentes mais « équivalentes ». La reconnaissance mutuelle de labels écologiques permettrait d'éviter ou d'atténuer les effets préjudiciables sur le commerce, tout en favorisant une promotion de la protection de l'environnement qui tiendrait compte des différences qui séparent les situations environnementales des pays concernés.

Depuis quelque temps, la reconnaissance mutuelle des systèmes de certification écologique suscite un intérêt accru. On craint notamment que la multiplication de labels écologiques dans un nombre croissant de pays ne porte préjudice au commerce et ne crée une certaine confusion dans l'esprit des consommateurs. Le principe de la reconnaissance mutuelle est toutefois loin d'être largement admis. Pour certains groupes de défense de l'environnement, il ne serait en effet pas acceptable que des produits qui ne satisfont pas aux critères rigoureux du système national bénéficient néanmoins du label écologique correspondant au nom, justement, de la reconnaissance mutuelle. De leur côté, les producteurs nationaux pourraient craindre une baisse de leur compétitivité. La reconnaissance mutuelle passe donc par l'instauration d'un climat de confiance. Par ailleurs, pour être bien accepté par les consommateurs et par les groupes d'intérêts écologiques, le système de certification du pays exportateur doit être parfaitement crédible.

${ }^{15}$ L'Accord sur les obstacles techniques au commerce stipule ce qui suit : « Les membres envisageront de manière positive d'accepter comme équivalents les règlements techniques des autres membres, même si ces règlements diffèrent des leurs, à condition d'avoir la certitude que ces règlements remplissent de manière adéquate les objectifs de leurs propres règlements. " (Art. 27)

${ }^{16}$ L'Accord sur l'application des mesures sanitaires et phytosanitaires comprend la disposition suivante : « Les membres accepteront les mesures sanitaires ou phytosanitaires d'autres membres comme équivalentes, même si ces mesures diffèrent des leurs ou de celles utilisées par d'autres membres s'occupant du commerce du même produit, si le pays exportateur démontre objectivement au membre importateur qu'avec ses mesures le niveau approprié de protection sanitaire ou phytosanitaire du membre importateur est atteint. " (Art. 14) 
La reconnaissance mutuelle présente un intérêt fondamental pour les pays en développement et constitue un important objectif à long terme dans le travail de mise en place de programmes de certification écologique dans certains de ces pays. Elle ne sert toutefois pas seulement des intérêts commerciaux. La reconnaissance mutuelle apparaît plutôt comme une condition essentielle qui garantit que les labels écologiques auront effectivement un impact positif sur l'environnement des pays en développement.

\subsection{LABELS ÉCOLOGIQUES ET OMC}

Pour éviter ou atténuer les effets préjudiciables que les labels écologiques pourraient avoir sur le commerce, il importe avant tout de renforcer la coopération internationale et d'accroître la transparence. Dans ce cadre, il serait utile de tirer au clair les liens qui existent entre les labels écologiques et l'OMC, plus particulièrement l'Accord sur les obstacles techniques au commerce. Contrairement à ce que l'on prétend souvent, le caractère facultatif de la certification écologique et le fait que sa gestion soit assurée par des organismes non gouvernementaux, ne signifie nullement que cette certification ne puisse pas être régie par l'Accord sur les obstacles techniques au commerce. Les normes facultatives appliquées par un gouvernement central, un gouvernement local, un organisme non gouvernemental ou par un organisme régional, sont en fait soumis au Code de pratique pour l'élaboration, l'adoption et l'application de normes (annexe 2 à l'Accord sur les obstacles techniques au commerce).

L'application de critères liés aux procédés et aux méthodes de production sans rapport avec le produit (portant un label écologique) pourrait toutefois rendre difficiles les relations entre la certification écologique et les règlements de l'OMC. D'aucuns pourraient en effet craindre qu'en soumettant les labels écologiques aux dispositions de l'Accord sur les obstacles techniques au commerce on créerait un précédent en matière de reconnaissance de procédés et de méthodes de production non prévues par l'OMC. Mais rien n'empêche la communauté internationale de négocier un code de certification écologique spécifique ou d'autres instruments facultatifs dans le cadre de l'OMC.

\section{CONCLUSIONS}

Les labels écologiques peuvent constituer un instrument efficace de la politique de l'environnement, à condition d'être non discriminatoires, transparents et fondés sur une procédure d'élaboration ouverte à tous, y compris - lorsque c'est indiqué - aux producteurs étrangers. Faute de quoi, la certification écologique peut aussi devenir un obstacle non tarifaire au commerce.

A certains égards, les labels écologiques ont sur le commerce des répercussions analogues à celles des normes et règlements techniques. Afin d'atténuer ces effets indésirables, on pourrait donc s'inspirer des mesures prises pour parer aux conséquences de ces dernières, par exemple dans le cadre de l'Accord sur les obstacles techniques au commerce.

Les labels écologiques pourraient aussi engendrer des effets que les règles du commerce international n'ont pas envisagés. L'analyse du cycle de vie et l'ap- 
plication de critères relatifs aux procédés et aux méthodes de production soulèvent ainsi des questions complexes, en particulier dans l'optique des échanges internationaux. Ces aspects de la certification écologique nécessitent une conception de la transparence qui soit généralement admise.

On escompte par ailleurs que les principes directeurs que l'ISO élabore actuellement contribuent beaucoup à la crédibilité des systèmes de certification écologique et qu'ils permettent d'éviter les discriminations. Les labels écologiques n'en continueront pas moins de susciter des inquiétudes, notamment dans les pays en développement, car nombre de questions restent encore sans réponse :

- Relations entre la certification écologique et les mécanismes du système commercial multilatéral, notamment l'Accord sur les obstacles techniques au commerce (ce point figure dans le mandat du Comité sur le commerce et l'environnement de l'OMC) ;

- Coûts de transaction pouvant découler de la coexistence de différents systèmes de certification écologique (relevons ici que le travail de l'ISO ne vise pas à fixer des critères uniformes de certification écologique qui seraient utilisés dans tous les systèmes) ;

- Critères liés aux procédés et aux méthodes de production ;

- Reconnaissance mutuelle et équivalences.

Le présent article a passé en revue plusieurs moyens de tenir compte des intérêts des producteurs étrangers, y compris de ceux des pays en développement, dans l'élaboration de critères de certification écologique. A court terme, il apparaît judicieux d'accroître la transparence et d'accorder une attention particulière aux critères liés aux procédés et aux méthodes de production pour les catégories de produits où les importations, notamment celles en provenance de pays en développement, occupent une grande place. A plus long terme, il serait indiqué d'explorer la voie des équivalences et de la reconnaissance mutuelle entre les divers systèmes de certification écologique. Le PNUE et la CNUCED collaborent pour résoudre les divers problèmes dans ce domaine. 